\title{
Fluorine-Substituted Phenanthro[9,10-d]imidazole Derivatives with Optimized Charge-Transfer Characteristics for Efficient Deep-Blue Emitters
}

\author{
Zhiqiang Li $\mathbf{i}^{\mathbf{a}}(\mathbb{D}$ \\ Ning $\mathrm{Xie}^{\mathrm{a}}$ \\ Yincai $\mathrm{Xu}^{\mathrm{a}}$ \\ Chenglong $\mathbf{L i}^{\mathrm{a}}$ (i) \\ Xiaoyue $\mathrm{Mu}^{* a}$ (i) \\ Yue Wang*a (i)

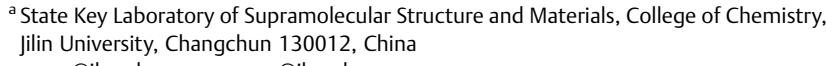 \\ xymu@jlu.edu.cn; yuewang@jlu.edu.cn
}

Received: 15.10 .2019
Accepted after revision: 19.11 .2019

DOI: 10.1055/s-0039-3402513; Art ID: om-19-0019-oa

License terms: (c)

(c) 2020. The Author(s). This is an open access article published by Thieme under the terms of the Creative Commons Attribution-NonDerivative-NonCommercial-License, permitting copying and reproduction so long as the original work is given appropriate credit. Contents may not be used for commercial purposes, or adapted, remixed, transformed or built upon. (https://creativecommons.org/licenses/by-nc-nd/4.0/).

Abstract The development of high-efficiency deep-blue emitters is of great importance for full-color organic light-emitting diodes (OLEDs). In this contribution, three difluorine-substituted phenanthro[9,10-d]imidazole derivatives with optimized charge-transfer character and deep-blue emission have been developed. It is demonstrated that the fluorine substitution can facilitate the "state mixing" of singlet and triplet excitons, which increases the utilization of triplet excitons. The fluorine substitution also brings more intermolecular interactions which have influence on the molecular packing pattern of the solid states, ultimately impacting their carrier mobilities. Through fine-tuning of molecular structures, 4'-(1-(3,5difluorophenyl)-1H-phenanthro[9,10-d]imidazol-2-yl)-N,N-diphenyl[1,1'-biphenyl]-4-amine (2FPPIDPA) realized a high exciton utilization ratio with Commission Internationale de L'Eclairage (CIE) coordinates of $(0.156$, $0.046)$, and 4'-(1-(3,5-difluorophenyl)-1H-phenanthro[9,10-d]imidazol2-yl)-N,N-diphenyl-[1,1'-biphenyl]-4-amine (2FPPIDPA) achieved an external quantum efficiency of $8.47 \%$ with $\mathrm{CIE}$ coordinates of $(0.152,0.083)$ in multilayer OLEDs. Due to their good hole-transport abilities, doublelayer OLEDs without the hole-transport layer showed performances comparable or even superior to the multilayer ones.

Key words organic light-emitting diodes, blue emitters, phenanthro [9, 10-d]imidazole, charge-transfer state, fluorine substituents

\section{Introduction}

Blue, especially deep-blue, emitters are crucial for the development of organic light-emitting diodes (OLEDs) not only because they can act as hosts for other color materials, ${ }^{1}$

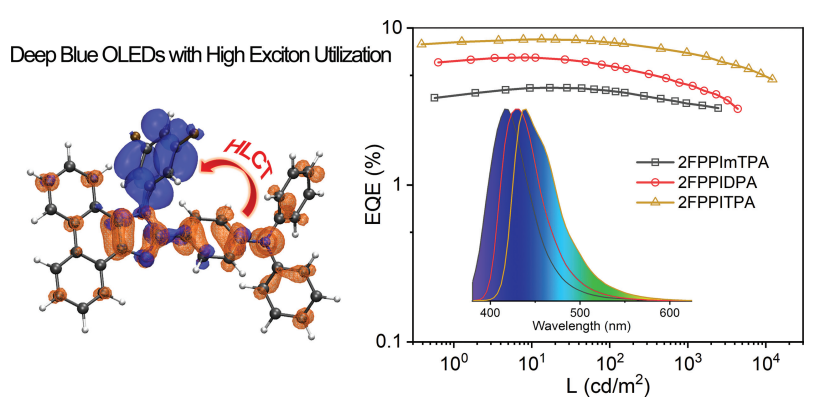

but also they can be directly used as luminescent materials to reduce the power consumptions of full-color OLEDs. ${ }^{2}$ In order to achieve high external quantum efficiency (EQE), a large number of high-performance blue phosphorescent and thermally activated delayed fluorescence (TADF) materials have been exploited. However, the severe efficiency roll-offs induced by long exciton lifetimes and low maximum luminance strictly confine their applications. $^{3}$ To make things worse, TADF and phosphorescent emitters always show broad emission spectra due to the intramolecular charge-transfer state (ICT) or metal-toligand charge transfer (MLCT) character. ${ }^{4}$ Consequently, only few TADF and phosphorescent OLEDs can meet the blue standard of Commission International de L'Eclairage (CIE) chromaticity coordinate of $(0.15,0.06)$ required for the International Telecommunication Union Recommendation (ITU-R) BT.709. Therefore, the development of efficient deep-blue emitters is still an urgent need.

Apart from TADF and phosphorescent mechanisms, Ma and Yang's group put forward the hybridized local and chargetransfer (HLCT) state mechanism that can also achieve 100\% electricity-generated exciton utilization. ${ }^{5}$ In HLCT materials, the exciton in the triplet state $T_{m}(m \geq 2)$ can convert into the singlet state $S_{n}(n \geq 1)$ through inverse intersystem crossing. Based on this mechanism, many blue emitters have been developed. In 2012, they reported a TPA-PPI-based device with CIE coordinates of $(0.15,0.11)$ and a maximum EQE of $5.02 \%{ }^{6}$ Not long after that they demonstrated that introducing a strong electron-withdrawing cyano group to the nitrogen-substituted benzene ring of TPM can effectively improve exciton utilization efficiency (EUE). ${ }^{7}$ Based on the above results, Yang's group developed cyano-substituted TPA-PPI, i.e., TBPMCN, which achieves an EQE of 7.8\%, an EUE of $97 \%$, and a CIE of $(0.16,0.16) .^{8}$ Recently, by introducing anthracene as a bridge between the donor and acceptor units in PAC, Ma et al. realized the first OLED based on the HLCT mechanism with over $10 \%$ EQE. $^{9}$ Besides these high- 
performance emitters, a variety of excellent OLEDs utilizing HLCT host materials have been reported, ${ }^{10}$ proving the versatility of HLCT molecules.

Although incorporating cyano or anthracene improves the performances of phenanthro[9,10-d]imidazole (PPI) derivative-based OLEDs, it also inevitably leads to poor color purities due to the enhanced charge-transfer (CT) state and electron coupling effects. Moreover, the strong CT states are usually accompanied by reduced fluorescence quantum yield $\left(\phi_{\mathrm{pl}}\right)$, which leads to low EQE. Aiming at solving the above issues, we introduced fluorine atoms into the two meta-positions of the nitrogen-substituted phenyl groups of PPI derivatives to delicately regulate the properties of the excited-state. Fluorine is the most electronegative element in the periodic table, and it has been proven that the fluorine atoms can alter energy levels without sacrificing bandgaps and improve molecular ordering by introducing supramolecular interactions. ${ }^{11}$ In this contribution we show how the HLCT state mixing status was greatly improved by introducing fluorine into PPI derivative. We also show that the corresponding nondoped OLEDs with deep-blue emissions were achieved and their performances were among the best of HLCT materials.

\section{Results and Discussion}

\section{Synthesis and Thermal Stability}

As shown in Scheme 1, all target molecules were synthesized within two facile steps with high yields (>70\%). PPI-brominated derivatives and 2FPPIDPA were synthesized through a one-pot cyclization reaction with phenanthraquinone, aromatic aldehydes, aromatic amine, and ammonium acetate, and then the brominated PPI reacted with 4- (diphenylamino)phenylboronic acid by the Suzuki coupling reaction to produce 2FPPImTPA and 2FPPITPA. The final products were further purified by a vacuum sublimation approach and fully characterized by ${ }^{1} \mathrm{H}$ NMR, mass spectrometry (ESI-ITMS), elemental analysis, and single-crystal X-ray analysis. Thermal stability of the target molecules was investigated through thermogravimetric analysis (TGA; Figure S1a) and differential scanning calorimetry (DSC; Figure S1b). All these materials show high decomposition temperatures ( $T_{\mathrm{d}}$ corresponding to $5 \%$ mass loss) of more than $417{ }^{\circ} \mathrm{C}$, suggesting excellent thermal stability in the device fabrication process by vacuum thermal deposition. The glass-transition temperatures $\left(T_{\mathrm{g}}\right)$ of 2FPPImTPA and 2FPPITPA are 118 and $131{ }^{\circ} \mathrm{C}$, respectively. High $T_{\mathrm{g}}$ is advantageous in avoiding crystallization of the material to maintain long-term stability of the device.

\section{Investigation of Fluorine Influences}

Firstly, the electronic structures of 2FPPImTPA, 2FPPIDPA, and 2FPPITPA were investigated by density functional theory (DFT) calculations. DFT calculations demonstrate that compared to their counterparts without fluorine substituents (Figure S2), the fluorine atoms cause more decrease in the LUMOs than the HOMOs (Figure 1). Furthermore, the fluorine atoms also induce more LUMO orbital distributions on the nitrogen-substituted phenyl groups of the PPI moiety, which brings more CT components to the $S_{0} \rightarrow S_{1}$ transitions as indicated by time-dependent DFT (TD-DFT) calculations. Reports reveal that increased CT components are beneficial to the "state mixing" of high-lying triplet states and singlet states. $^{8,12}$ To further explore their excited-state characteristics, "hole-electron" analyses of some transitions were

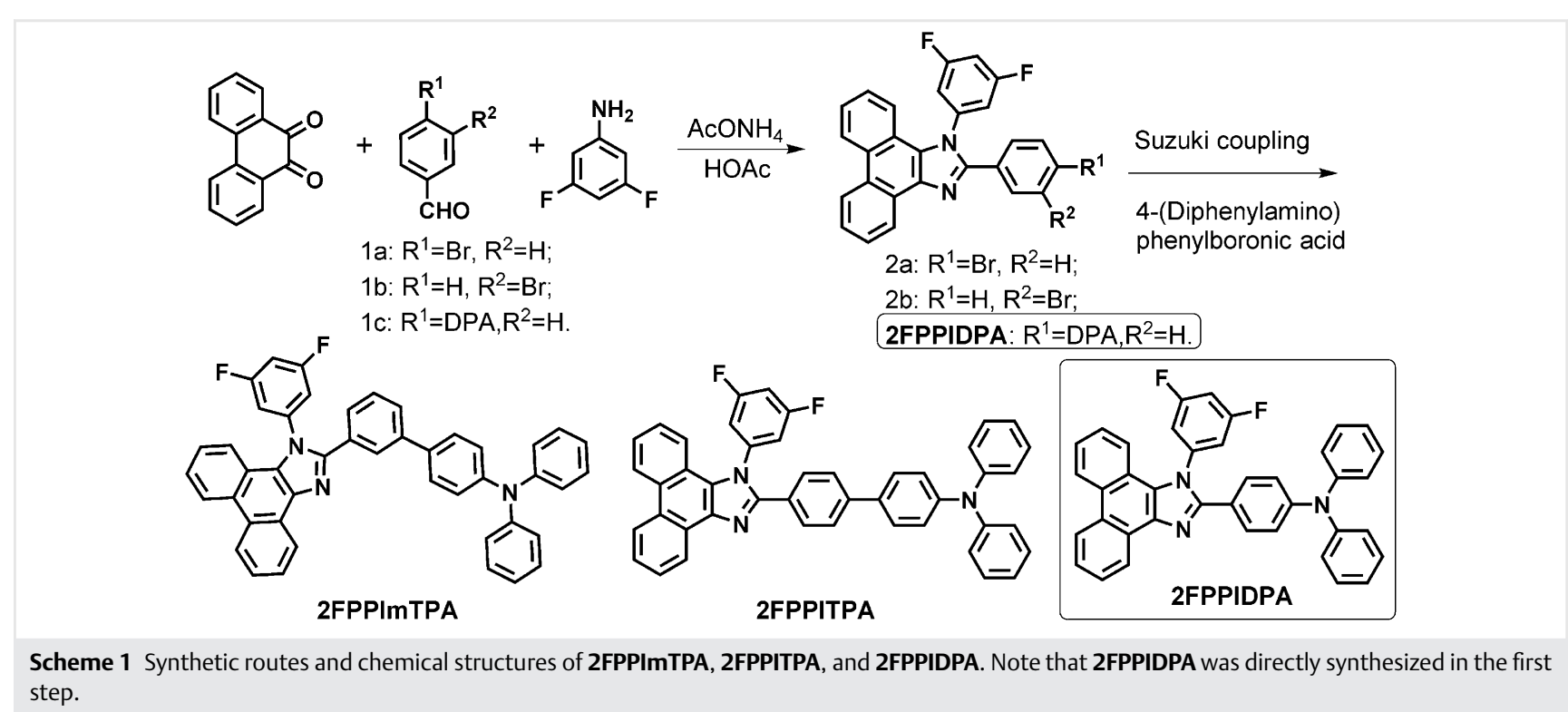



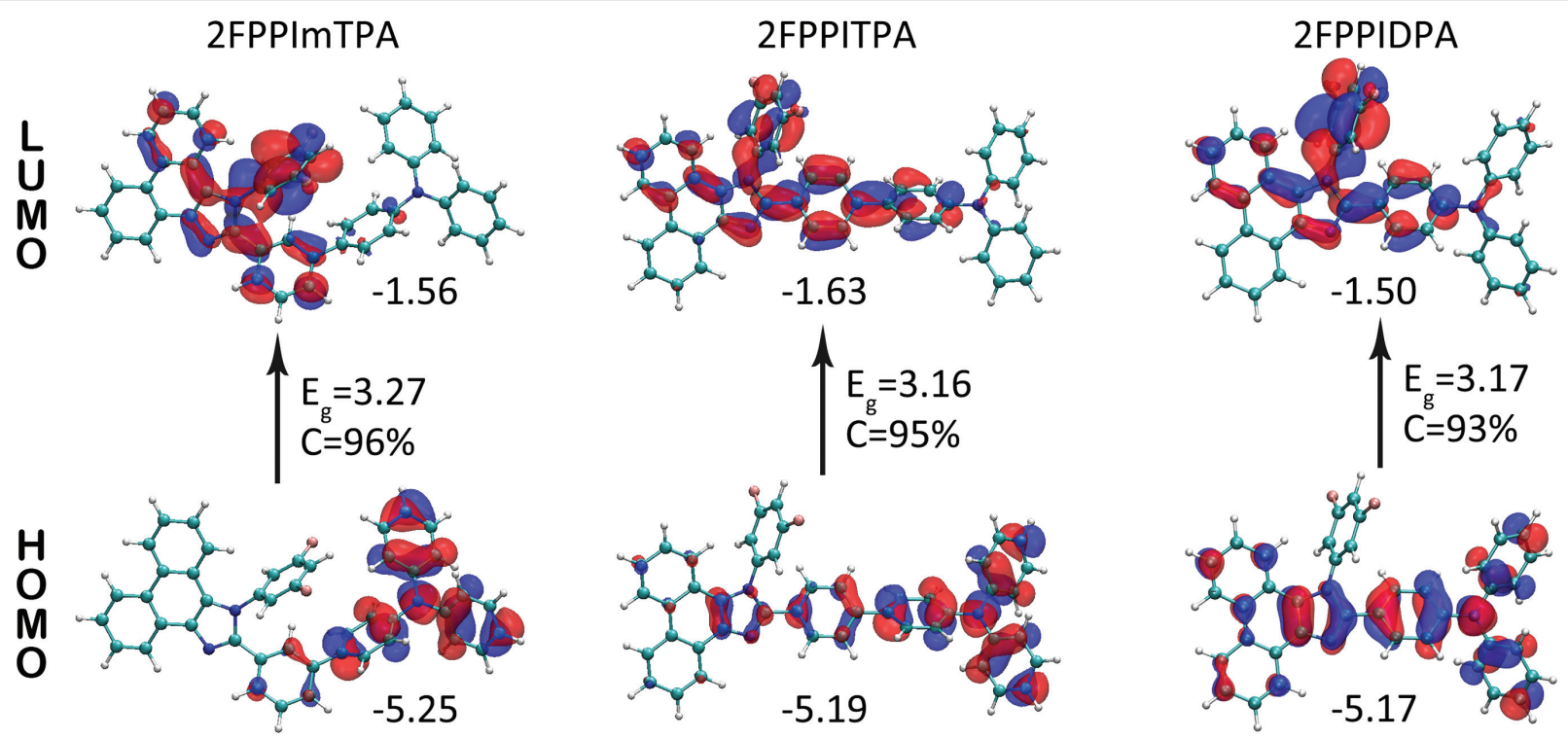

Figure 1 Frontier molecular orbital distributions and energy levels of target molecules calculated by B3LYP-D3/6-311(g, d). $E_{\mathrm{g}}$ represents the vertical transition energy based on $S_{0}$ geometries of $S_{0} \rightarrow S_{1}$ transition calculated by TD-DFT, and C represents the contribution ratios of HOMO $\rightarrow$ LUMO to $S_{0} \rightarrow S_{1}$ transitions. The unit of HOMO, LUMO, and $E_{\mathrm{g}}$ is $\mathrm{eV}$.

analyzed using Multiwfn 3.6, ${ }^{13}$ and the detailed information is given in Table S1, Table S2, and Figure S3. Different CT and locally excited (LE) components and distribution characteristics in $S_{1}$ and $T_{n}$ with similar energy (Figure $S 3$ ) are beneficial for the reverse intersystem crossing process according to the El-Sayed rule. ${ }^{14}$ From the "hole-electron" analyses we can see that the excited-state transitions of 2FPPImTPA, 2FPPIDPA, and 2FPPITPA are consistent with the characteristics of the HLCT state. The CT component of the $S_{0} \rightarrow S_{1}$ transition decreases while the LE component increases in the order of 2FPPImTPA, 2FPPIDPA, and 2FPPITPA as shown by the three indexes in Table S2. For 2FPPImTPA, the CT component is dominant, while for 2FPPITPA, the LE component is highlighted, and 2FPPIDPA displays a balanced LE and CT component ratio. More importantly, the energy bandgaps of $S_{0} \rightarrow S_{1}$ transitions drop negligibly $(<0.02 \mathrm{eV})$ for 2FPPIDPA and 2FPPITPA and only $0.2 \mathrm{eV}$ for 2FPPImTPA (see $E_{\mathrm{g}}$ values in Figures 1 and S2). Owing to the meta-connection which reduces the degree of conjugation, 2FPPImTPA is still the bluest emitter among the three molecules. Theoretical calculations distinctly confirm that the fluorine atoms located on the meta-positions of the nitrogen-substituted phenyl groups can effectively influence the excited-state properties of PPI derivatives, while maintaining deep-blue emission simultaneously.

\section{Crystal-Structure Analyses}

Single crystals were grown for these materials to investigate their molecular structures and packing modes.
In the 2FPPImTPA crystal (Figure 2a): C-H...F hydrogen bonds were formed between the hydrogen of triphenylamine (TPA) and the adjacent molecule fluorine at a distance of $2.593 \AA$; $C-H . . . \pi$ interactions assembled between the hydrogen of the difluorophenyl group and phenanthrene at a distance of $2.469 \AA$ and $\mathrm{C}-\mathrm{H} . . . \pi$ interactions between the hydrogen of the difluorophenyl group and phenylene bridge at a distance of $2.858 \AA$ were established. In the 2FPPIDPA crystal (Figure 2b), molecules are arranged layer by layer along the plane formed by the $a$ and $c$ crystallographic axes, and the molecules in the same layer are assembled in the same direction but those in adjacent layers are antiparallel. Two 2FPPIDPA molecules of adjacent layers formed "dimers" through mutual $\mathrm{C}-\mathrm{H}$... $\mathrm{N}$ hydrogen bonds (2.682 $\mathrm{A}$ ), and $\mathrm{C}-\mathrm{H}$....F hydrogen bonds formed between the hydrogen of phenanthrene and fluorine (2.647 $\AA$ ). In the 2FPPITPA crystal (Figure 2c), the $\mathrm{C}-\mathrm{H}$...F hydrogen bonds between the hydrogen of phenanthrene and fluorine are $2.598 \AA$ in length; there are also $\mathrm{C}-\mathrm{H} . . . \pi$ interactions linked between adjacent TPA groups $(2.772 \AA)$ and the hydrogen of the difluorophenyl group and phenanthrene $(2.506 \AA)$. The introduction of fluorine makes our PPI derivatives pile up more compact compared with their counterparts as indicated by their bigger crystal density (see Table S3, $\rho$ column), which will reduce the excited nonradiative decay by molecular vibration and increase $\phi_{\mathrm{pl}}$ in the solid state. ${ }^{15}$ Meanwhile, the twisted molecular structures resulting from the propeller TPA and nitrogen-substituted phenyl with large torsional angles are beneficial for suppressing the formation of excimers and exciplexes in the solid state. ${ }^{16}$ 


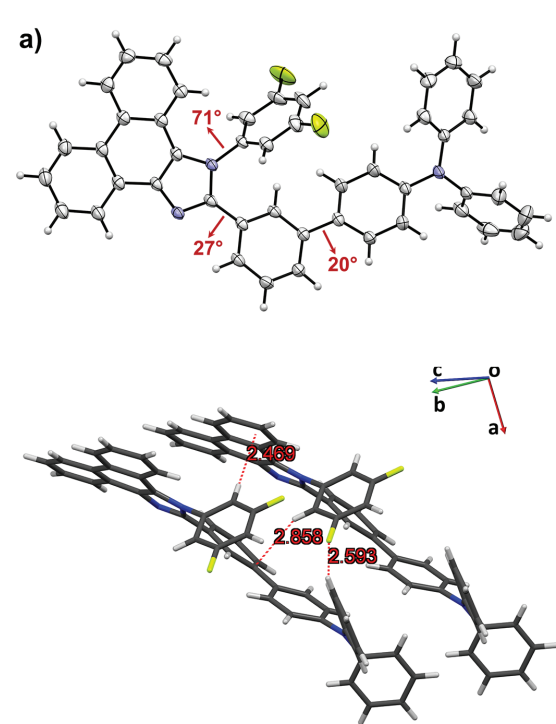

2FPPImTPA

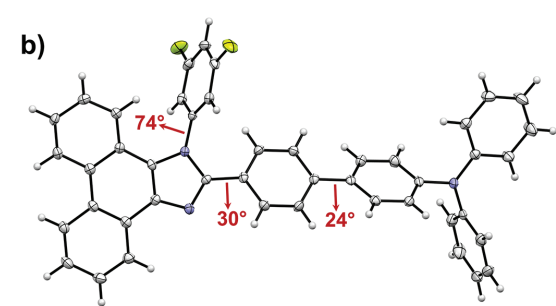<smiles>CC(C)C</smiles>

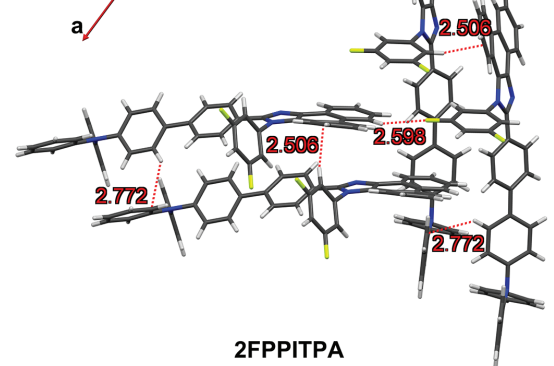

c)

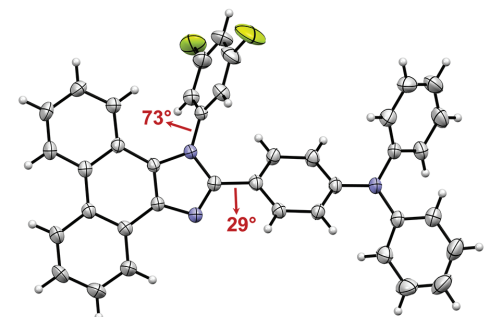

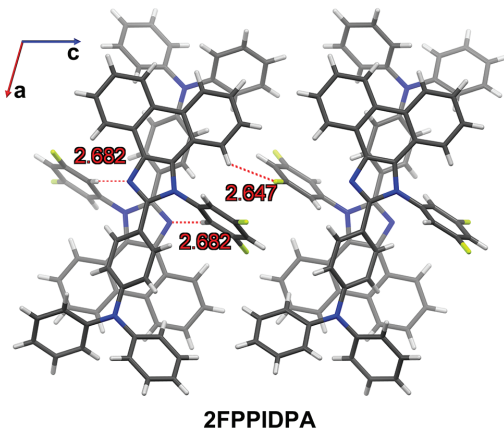

Figure 2 The oak ridge thermal ellipsoid plots (ORTEPs) with important dihedral angle marked and major intermolecular interactions in crystals of (a) 2FPPImTPA, (b) 2FPPIDPA, and (c) 2FPPITPA.

\section{Photophysical Properties}

UV-visible absorption spectra and photoluminescence (PL) spectra of all target molecules in dilute dichloromethane solution $\left(10^{-5} \mathrm{~mol} \mathrm{~L}^{-1}\right)$ are shown in Figure 3. 2FPPITPA and 2FPPIDPA show similar absorption spectra, and their longest-wavelength absorption peaks are at $363 \mathrm{~nm}$, which are attributed to the $\mathrm{S}_{0} \rightarrow \mathrm{S}_{1}$ transition with the mixing of CT and LE state as revealed by TD-DFT. 2FPPImTPA also has a shoulder peak at $358 \mathrm{~nm}$ with a relatively lower extinction coefficient, which is attributed

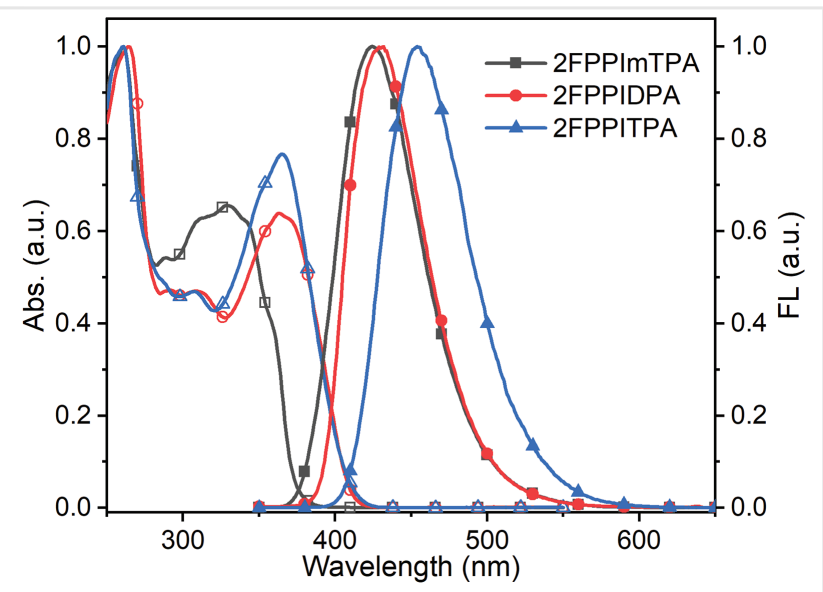

Figure 3 UV-visible absorption spectra and fluorescence spectra measured in dichloromethane. mainly to the CT characteristics resulting from the metasubstitution of TPA to PPI. Absorption peaks in the 300$335 \mathrm{~nm}$ range are attributed to the $\pi-\pi^{*}$ transitions of PPI moieties. The absorption spectra of their films are the same as the solutions (Figure S4).

Although introduction of fluorine induces stronger CT properties, these molecules all showed deep-blue emissions both in solutions and in nondoped films, which is in good agreement with the DFT results. Some key physical properties of the target molecules are listed in Table 1. The triplet energy levels of these molecules are all about $2.4 \mathrm{eV}$, estimated from the highest-energy-vibration subbands of phosphorescence $(\mathrm{PH})$ spectra in toluene at $77 \mathrm{~K}$ (Figure S5). The fluorescence quantum yields $\left(\phi_{\mathrm{pl}}\right)$ of all

Table 1 Summary of key physical properties

\begin{tabular}{llllll}
\hline & $\begin{array}{l}\lambda_{\mathrm{em}}{ }^{\mathrm{a}} \\
{[\mathrm{nm}]}\end{array}$ & $\begin{array}{l}E_{\mathrm{T}}^{\mathrm{b}} \\
{[\mathrm{eV}]}\end{array}$ & $\begin{array}{l}\mathrm{HOMO} / \mathrm{LUMO} \\
{[\mathrm{eV}]^{\mathrm{c}}}\end{array}$ & $\begin{array}{l}\phi_{\mathrm{pl}} \\
{[\%]^{\mathrm{d}}}\end{array}$ & $\begin{array}{l}T_{\mathrm{g}}{ }^{\mathrm{e}} / T_{\mathrm{d}}{ }^{\mathrm{f}} \\
{\left[{ }^{\circ} \mathrm{C}\right]}\end{array}$ \\
\hline 2FPPImTPA & $424 / 405$ & 2.48 & $-5.3 /-2.2$ & $71 / 35$ & $118 / 441$ \\
2FPPIDPA & $430 / 433$ & 2.36 & $-5.2 /-2.2$ & $76 / 33$ & $\mathrm{no} / 417$ \\
2FPPITPA & $453 / 461$ & 2.40 & $-5.2 /-2.3$ & $90 / 52$ & $131 / 457$ \\
\hline
\end{tabular}

aEmission maximum in dichloromethane (left) and in nondoped film (right). ${ }^{\mathrm{b}}$ The triplet energy levels were estimated from the highest-energy-vibration sub-bands of PL spectra in toluene at $77 \mathrm{~K}$.

CHOMO and LUMO levels were measured by cyclic voltammetry based on the oxidation potential of ferrocene $(4.8 \mathrm{eV})$ as the reference.

${ }^{\mathrm{d} F l u o r e s c e n c e ~ q u a n t u m ~ y i e l d ~ i n ~ d i c h l o r o m e t h a n e ~ s o l u t i o n ~(l e f t) ~ a n d ~ i n ~ f i l m ~ s t a t e ~}$ (right).

'The glass-transition temperatures.

The thermal decomposition temperatures corresponding to $5 \%$ weight loss. 
target molecules are very high in dichloromethane solution, which are 71\% for 2FPPImTPA, 76\% for 2FPPIDPA, and 90\% for 2FPPITPA, respectively. However, their $\phi_{\mathrm{pl}}$ values in the film state are only about half of those in solution, resulting from an aggregation-caused quenching effect. ${ }^{17}$ 2FPPITPA has a higher $\phi_{\mathrm{pl}}$ than the other two molecules, which originates from the increased degree of conjugation and frontier orbital overlap (Table S2).

In order to further explore the luminescence properties, solvation effect tests of all target molecules were carried out, and the results are listed in Figure S6. Their visible absorption spectra show nearly no change in different solvents, while their fluorescence spectra showed a significant redshift when solvent polarities were increased [e.g., hexane to dimethyl sulfoxide (DMSO)]. The redshift of 2FPPIDPA (41 nm) is smaller than those of 2FPPITPA and 2FPPImTPA ( $>65 \mathrm{~nm}$ ), resulting from the short CT distance of 2FPPIDPA as revealed by the "hole-electron" analyses ( $D$ index in Table S2). In addition, the fluorescence spectra of 2FPPITPA and 2FPPIDPA exhibit fine vibrational structures with LE properties in low polarity solvents, but are structureless with CT properties in high polarity solvents, which attests again to their HLCT characteristics. ${ }^{18}$

\section{Energy Levels and Single-Carrier Devices}

Before applying our materials to OLEDs, their electrochemical energy levels were investigated by cyclic voltammetry using ferrocene as a reference (Figure S7). The HOMOs of these materials are all about $-5.2 \mathrm{eV}$, which is attributed to the oxidation of the TPA group, and their LUMOs are all about $-2.2 \mathrm{eV}$, which is attributed to the reduction of the PPI group. ${ }^{19}$ To check the carrier-transporting properties of 2FPPImTPA, 2FPPITPA, and 2FPPIDPA, single-carrier devices with the structures of [indium-tin oxide (ITO)/1,4-bis[(1-naphthylphenyl)amino]biphenyl (NPB) $(10 \mathrm{~nm}) /$ target materials $(80 \mathrm{~nm}) / \mathrm{NPB}$ $(10 \mathrm{~nm}) / \mathrm{Al}(100 \mathrm{~nm})]$ for hole-only devices, and [ITO/ 1,3,5-tris(N-phenylbenzimidazol-2-yl) benzene (TPBi) $(10 \mathrm{~nm}) /$ target materials $(80 \mathrm{~nm}) / \mathrm{TPBi} \quad(10 \mathrm{~nm}) / \mathrm{LiF}$ $(1 \mathrm{~nm}) / \mathrm{Al}(100 \mathrm{~nm})]$ for electron-only devices were fabricated. Here, NPB and TPBi are used to prevent electron and hole injection from the cathode and anode, respectively. Figure 4 depicts the current density-voltage plots of all our fabricated single-carrier devices and comparison of NPB hole-only devices, from which we can see that as the molecular conjugates increase, the difference in their electron and hole transport abilities decrease. Since PPI itself is a bipolar transport material with similar electron and hole transport capabilities, ${ }^{19 b}$ the incorporation of TPA with good hole transport ability enhances hole mobility over electron mobility for all target materials. Intriguingly, the hole transport abilities of our target materials are

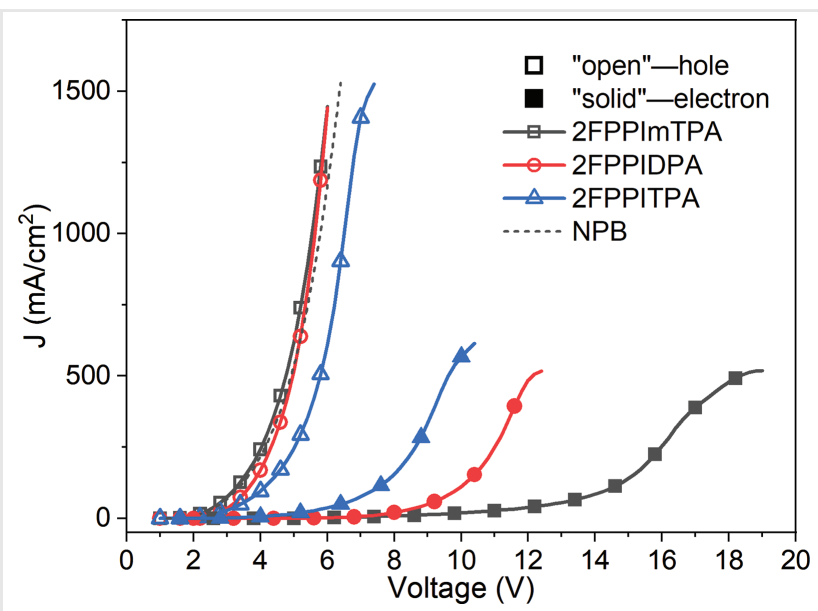

Figure 4 The current density-voltage $(U-V)$ plots of all single-carrier devices. The dash line represents a comparison of NPB hole-only device with a structure of [ITO/NPB $(100 \mathrm{~nm}) / \mathrm{Al}(100 \mathrm{~nm})]$.

approximately equal to that of NPB or even better than NPB, which may have resulted from the compact packing of these fluorine-substituted PPI derivatives.

\section{OLED Performances}

We fabricated multilayer nondoped OLEDs via a vacuum evaporation technique with the device structure of [ITO/NPB $(40 \mathrm{~nm}) /$ TCTA $(5 \mathrm{~nm}) /$ emitting layer $(20 \mathrm{~nm}) / \mathrm{TPBi}(35 \mathrm{~nm}) /$ $\mathrm{LiF}(0.8 \mathrm{~nm}) / \mathrm{Al}(100 \mathrm{~nm})]$. TCTA stands for 4,4',4"-tris(carbazol-9-yl)triphenylamine. Since the hole transport capability of our material is far superior to the corresponding electron transport capability, TCTA acted as a block layer to slow the hole mobility and was utilized for its deeper HOMO, which can further block the excitons due to its high triplet energies and broad bandgap. ${ }^{20}$ The energy-level diagrams and molecular structures of the materials used in these devices are shown in Figure S8 and the performances of these multilayer OLEDs are depicted in Figure 5 and Table 2. These OLEDs have very low turn-on voltages of no more than $3 \mathrm{~V}$, resulting from low carrier-injection barriers. The peaks of their electroluminescence (EL) spectra are below $440 \mathrm{~nm}$ and are located in the deep-blue region. The CIE coordinates of 2FPPImTPA and 2FPPIDPA devices are $(0.160,0.038)$ and $(0.156,0.046)$, respectively, which can meet the ITU-R BT.709 blue standard without any color filter. The EQEs of 2FPPImTPA, 2FPPIDPA, and 2FPPITPA multilayer devices are 4.12, 6.49, and 8.47\%, respectively, which are far superior to those of their counterparts (Table S3) and are among the best of deep-blue materials with similar CIE coordinates. ${ }^{19 b, 21}$ What's more, the efficiency roll-offs are less than $20 \%$ at $1000 \mathrm{~cd} \mathrm{~m}^{-2}$ for 2FPPImTPA and 2FPPITPA devices, since there are no detrimental intermolecular interactions as indicated by the molecule packing modes 

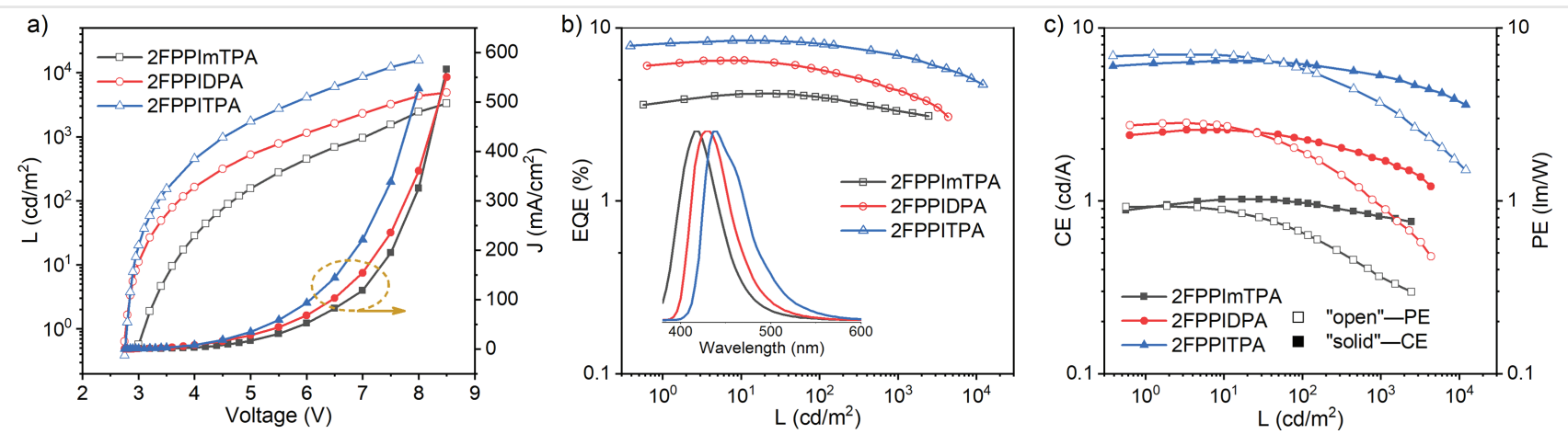

Figure 5 (a) Current density-voltage-luminance (J-V-L) plots, (b) EQEs versus luminance curves with EL spectra at $100 \mathrm{~cd} \mathrm{~m}^{-2}$ as an inset, and (c) power efficiencies (PEs) and current efficiencies (CEs) versus luminance curves for multilayer OLEDs.

Table 2 Summary of OLED performances in this study

\begin{tabular}{llllllll}
\hline Emitter & $\begin{array}{l}V_{\text {on }} \\
{[\mathrm{V}]^{\mathrm{a}}}\end{array}$ & $\begin{array}{l}\lambda_{\mathrm{em}} \\
{[\mathrm{nm}]}\end{array}$ & $\begin{array}{l}L_{\max } \\
{\left[\mathrm{cd} \mathrm{m}^{-2}\right]^{\mathrm{b}}}\end{array}$ & $\begin{array}{l}\mathrm{PE}_{\max } \\
{\left[\mathrm{Im} \mathrm{W}^{-1}\right]^{\mathrm{c}}}\end{array}$ & $\begin{array}{l}\mathrm{CE}_{\max } \\
\left.[\mathrm{cd} \mathrm{A}]^{-1}\right]^{\mathrm{d}}\end{array}$ & $\begin{array}{l}\mathrm{CIE} \\
(x, y)^{\mathrm{e}}\end{array}$ & $\begin{array}{l}\mathrm{EQE} \\
{[\%]^{\mathrm{max} / 1000}}\end{array}$ \\
\hline 2FPPImTPA$(m)$ & 3.0 & 416 & 15560 & $0.93 / 0.36$ & $1.10 / 0.81$ & $0.160,0.038$ & $4.12 / 3.30$ \\
2FPPIDPA $(m)$ & 2.8 & 430 & 4916 & $2.83 / 0.93$ & $2.58 / 1.72$ & $0.156,0.046$ & $6.49 / 4.36$ \\
2FPPITPA $(m)$ & $2 . .8$ & 440 & 3798 & $7.00 / 3.66$ & $6.47 / 5.30$ & $0.152,0.083$ & $8.47 / 6.90$ \\
2FPPImTPA(d) & 3.0 & 404 & 1417 & $0.93 / 0.27$ & $0.99 / 0.55$ & $0.159,0.047$ & $4.10 / 2.32$ \\
2FPPIDPA(d) & 2.8 & 420 & 3822 & $3.40 / 0.93$ & $3.02 / 1.80$ & $0.156,0.055$ & $6.73 / 4.01$ \\
2FPPITPA $(d)$ & 2.8 & 440 & 13870 & $6.64 / 4.28$ & $6.55 / 5.94$ & $0.154,0.128$ & $6.10 / 5.54$ \\
\hline
\end{tabular}

Note: The $m$ in parentheses represents the multilayer device structure, and $d$ represents the double-layer device structure.

aTurn-on voltage at $1 \mathrm{~cd} \mathrm{~m}^{-2}$.

bThe maximum luminance.

'The maximum power efficiency and power efficiency at $1000 \mathrm{~cd} \mathrm{~m}^{-2}$.

${ }^{\mathrm{d}}$ The maximum current efficiency and current efficiency at $1000 \mathrm{~cd} \mathrm{~m}{ }^{-2}$.

${ }^{\mathrm{e}} \mathrm{CIE}$ coordinates recorded at $100 \mathrm{~cd} \mathrm{~m}^{-2}$.

${ }^{\mathrm{f}}$ The maximum EQEs and EQEs at $1000 \mathrm{~cd} \mathrm{~m}^{-2}$.

in crystals (Figure 2a, b). However, the efficiency roll-off of 2FPPIDPA is $33 \%$, which is attributed to the antiparallel "dimer"-like packing mode that can lead to intermolecular CT (Figure 2c).22

Conventionally, the EQE of OLEDs can be expressed by the following equation ${ }^{23}$ :

$$
\mathrm{EQE}=\eta_{\mathrm{eu}} \times \gamma \times \eta_{\mathrm{out}} \times \phi_{\mathrm{pl}}
$$

(Equation 1)

where $\eta_{\mathrm{eu}}$ is the EUE for singlet and triplet excitons to generate photons, $\gamma$ is the carrier balance ratio of holes and electrons, which reaches close to unity for a multilayer device, and $\eta_{\text {out }}$ is the light out-coupling efficiency, which is considered to be about $20-30 \%$ without additional external light-extracting strategy. ${ }^{24}$ Assuming a $\eta_{\text {out }}$ of $30 \%$, the EQE of 2FPPITPA with the highest $\phi_{\mathrm{pl}}$ (52\% in nondoped film) is only $3.9 \%$ according to Equation 1. Therefore, the $\eta_{\mathrm{eu}}$ of 2FPPImTPA, 2FPPIDPA, and 2FPPITPA devices exceeds the $25 \%$ limit of traditional fluorescent materials. ${ }^{25}$ Since these molecules have a linear molecular configuration, they tend to accumulate parallel to the substrate with a horizontal transition dipole moment, which is conducive to the increase of $\eta_{\text {out }}$. The transition-dipole anisotropies of these molecules in nondoped films were investigated by florescence polarization experiments as described in our previous study. ${ }^{19 \mathrm{~b}}$ As shown in Figure S9, the emission intensity of the sample film excited by a fixed-intensity laser source gradually changed upon rotation of the polarizer, which indicates anisotropy of their transition dipole. Ratios of maximum-to-minimum emission intensity of 2FPPImTPA, 2FPPIDPA, and 2FPPITPA are $1.23,1.62$, and 1.47, respectively. 2FPPITPA possesses the strongest transition dipole anisotropy and the highest $\phi_{\mathrm{pl}}$ among these three materials, which explains why it has the highest EQE. However, both $\phi_{\mathrm{pl}}$ values and ratios of maximum-to-minimum emission intensity of these materials are smaller compared to those in our previous report, ${ }^{19 \mathrm{~b}}$ but their EQEs are very close, indicating that these materials have higher exciton utilization ratios. The higher exciton utilization ratios originate from the HLCT mechanism of these materials, which has been demonstrated in the above analyses. The $\eta_{\text {eu }}$ of 2FPPIDPA is about $66 \%$ assuming a $\eta_{\text {out }}$ of $30 \%$, which is attributed to the molecular-structure fine- 


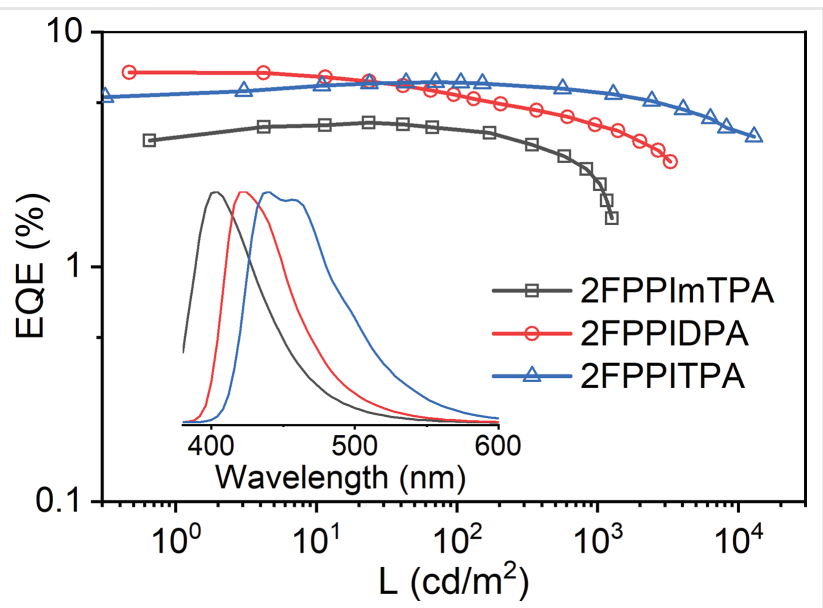

Figure 6 The EQE versus luminance curves with EL spectra at $100 \mathrm{~cd} \mathrm{~m}^{-2}$ as an inset for the double-layer OLEDs.

tuning resulted LE and CT component ratio equilibrium revealed by the "hole-electron" analyses. The high $\eta_{\mathrm{eu}}$ values of these materials manifest our success in improving the HLCT state mixing by fluorine substitution.

Encouraged by the excellent OLED performances and outstanding hole transport ability, we also fabricated simplified double-layer nondoped OLEDs without the hole-transport layer. The structure of the double-layer OLEDs is [ITO/emitting layer $(50 \mathrm{~nm}) / \mathrm{TPBi}(35 \mathrm{~nm}) / \mathrm{LiF}(0.8 \mathrm{~nm}) / \mathrm{Al}$ $(100 \mathrm{~nm})]$; the performances of these double-layer OLEDs are also listed in Table 2. Figure 6 shows the EQEversus luminance curves with EL spectra as an inset for these double-layer OLEDs, and other information is shown in Figure S10. The spectrum profiles and the peaks of their EL spectra for these double-layer devices are similar to those of their multilayer OLEDs, proving that they all originate from the luminescence of our target molecules. The maximum power efficiencies (PEs) and maximum current efficiencies (CEs) of 2FPPImTPA and 2FPPITPA double-layer devices are nearly the same as their multilayer OLEDs, but their maximum EQEs decreased due to the increase of spectrum full-width at half-maximum (FWHM). Surprisingly, the 2FPPIDPA double-layer device performance exceeded its corresponding multilayer OLED, which implies a huge potential for its application in OLEDs with simple device structures.

\section{Conclusions}

In summary, three molecules composed of fluorinesubstituted PPI and TPA with different degrees of conjugation were designed and synthesized, and the effects of chemical-structure changes on molecular luminescence properties and crystal packing patterns were fully studied. DFT calculations reveal that the introduction of fluorine brings more CT components to the $S_{0} \rightarrow S_{1}$ transitions of these molecules, which does not change the energy much but facilitates the "state mixing" of the HLCT state. Meanwhile, tailoring of the molecular structures through modifying the linking strategy can also change the LE and CT component ratio, and brings 2FPPIDPA to a balance of this ratio. Crystal analyses demonstrate that the fluorine substitution makes these molecules pile up more compact in the solid state, giving them high hole mobilities that are approximately equal to or even better than that of NPB. The nondoped multilayer OLEDs exhibit high EQE values of 4.12\% for 2FPPImTPA, $6.49 \%$ for 2FPPIDPA, and $8.47 \%$ for 2FPPITPA with deep-blue emissions, and their CIEs are $(0.160,0.038),(0.156,0.046)$, and $(0.152,0.083)$, respectively. Furthermore, the double-layer OLEDs without holetransport materials displayed comparable performances to their multilayer ones for 2FPPImTPA and 2FPPITPA, and a better performance for 2FPPIDPA. These results are among the best observed for nondoped deep-blue OLEDs. Our work shows that regulating the luminescence and accumulation characteristics of fluorine-substituted molecules can greatly enhance their properties, creating opportunity to produce highly efficient OLED materials.

\section{Experimental Section}

All reagents were bought from Energy Chemical Co. and/or JEK scientific Ltd. Co. and immediately used without further purification. The Schlenk technique was strictly performed under a nitrogen atmosphere in all reactions. The synthesis process is shown below in detail. The final products were first purified by column chromatography, then further purified by temperature-gradient vacuum sublimation.

\section{Procedures}

Reaction I: this is the general information for cyclization reactions. Phenanthrene-9,10-dione (1 eq.), 3,5-difluoroaniline ( 3 eq.), aromatic aldehyde (1.06 eq.), and ammonium acetate ( 5 eq.) were dissolved in acetic acid and refluxed in a nitrogen atmosphere for 6 hours. After cooled to room temperature, the system was poured into an equal volume of water and then separated by vacuum suction filtration. The filter cake was purified via column chromatography using dichloromethane and ethyl acetate as the eluent.

Reaction II: this is the general information for Suzuki coupling reactions. PPI-brominated derivatives (1 eq.), (4(diphenylamino)phenyl)boronic acid (1.1 eq.), $\mathrm{Pd}\left(\mathrm{PPh}_{3}\right)_{4}$ (0.04 eq.), $2 \mathrm{~mol} \mathrm{~L}^{-1} \mathrm{~K}_{2} \mathrm{CO}_{3}$ (3 eq.), tetrabutylammonium bromide (TBAB, 0.1 eq.), and toluene were refluxed in a nitrogen atmosphere for 12 hours. After cooled to room temperature, the system was poured into water and 
extracted with dichloromethane. Then the crude product was purified via column chromatography using dichloromethane and ethyl acetate as the eluent.

2a: phenanthrene-9,10-dione (1.25 g, $6 \mathrm{mmol}), 3,5-$ difluoroaniline (2.32 g, $18 \mathrm{mmol}), 1 \mathrm{a}(1.18 \mathrm{~g}, 6.36 \mathrm{mmol})$, and ammonium acetate $(2.31 \mathrm{~g}, 30 \mathrm{mmol})$ were used as reactants according to Reaction I to give 2a. A white powder, mass: 2.39 g, yield: $82 \% .{ }^{1} \mathrm{H}$ NMR (500 MHz, chloroform-d): $\delta=8.79$ (ddd, $J=18.8,8.2,1.3 \mathrm{~Hz}, 2 \mathrm{H}), 8.73-8.65(\mathrm{~m}, 1 \mathrm{H})$, 7.75 (ddd, $J=8.0,7.0,1.2 \mathrm{~Hz}, 1 \mathrm{H}$ ), 7.67 (ddd, $J=8.4,7.0$, $1.5 \mathrm{~Hz}, 1 \mathrm{H}$ ), 7.55 (ddd, $J=8.4,7.0,1.3 \mathrm{~Hz}, 1 \mathrm{H}$ ), 7.51-7.39 $(\mathrm{m}, 4 \mathrm{H}), 7.35$ (ddd, $J=8.3,7.0,1.2 \mathrm{~Hz}, 1 \mathrm{H}$ ), 7.20 (dd, $J=8.4,1.3 \mathrm{~Hz}, 1 \mathrm{H}$ ), 7.17-7.05 (m, $3 \mathrm{H}$ ). ESI-ITMS, $m / z$ : 484.32 and $486.33\left[\mathrm{M}^{+}\right]$. Calcd: 484.04 and 486.04 .

2b: phenanthrene-9,10-dione (1.25 g, $6 \mathrm{mmol})$, 3,5difluoroaniline (2.32 g, $18 \mathrm{mmol}), \mathbf{1 b}(1.18 \mathrm{~g}, 6.36 \mathrm{mmol})$, and ammonium acetate $(2.31 \mathrm{~g}, 30 \mathrm{mmol})$ were used as reactants according to Reaction I to give $\mathbf{2 b}$. A white powder, mass: 2.30 g, yield: $79 \% .{ }^{1} \mathrm{H}$ NMR (500 MHz, DMSO- $d_{6}$ ): $\delta=8.96(\mathrm{~d}, J=8.4 \mathrm{~Hz}, 1 \mathrm{H}), 8.90(\mathrm{~d}, J=8.3 \mathrm{~Hz}, 1 \mathrm{H}), 8.69$ $(\mathrm{dd}, J=8.0,1.4 \mathrm{~Hz}, 1 \mathrm{H}), 7.86-7.66(\mathrm{~m}, 6 \mathrm{H}), 7.66-7.59(\mathrm{~m}, 2$ H), 7.54 (dt, $J=7.8,1.3 \mathrm{~Hz}, 1 \mathrm{H}$ ), 7.51-7.45 (m, $1 \mathrm{H}), 7.38$ (t, $J=7.9 \mathrm{~Hz}, 1 \mathrm{H}$ ), 7.19 (dd, $J=8.3,1.2 \mathrm{~Hz}, 1 \mathrm{H}$ ). ESI-ITMS, $\mathrm{m} / z$ : 484.30 and $486.31\left[\mathrm{M}^{+}\right.$]. Calcd: 484.04 and 486.04 .

2FPPIDPA: phenanthrene-9,10-dione (1.25 g, $6 \mathrm{mmol}$ ), 3,5-difluoroaniline $(2.32 \mathrm{~g}, 18 \mathrm{mmol}), 1 \mathrm{c} \quad(1.74 \mathrm{~g}$, $6.36 \mathrm{mmol})$, and ammonium acetate $(2.31 \mathrm{~g}, 30 \mathrm{mmol})$ were used as reactants according to Reaction I to give 2FPPIDPA. A white powder, mass: $2.51 \mathrm{~g}$, yield: $73 \%{ }^{1} \mathrm{H}$ NMR (500 MHz, methylene chloride- $\left.d_{2}\right): \delta=8.86$ (d, $J=8.4 \mathrm{~Hz}, 1 \mathrm{H}), 8.79(\mathrm{~d}, J=8.3 \mathrm{~Hz}, 1 \mathrm{H}), 7.84(\mathrm{~s}, 1 \mathrm{H})$, 7.77 (s, $1 \mathrm{H}), 7.60$ (d, J = 46.5 Hz, $4 \mathrm{H}), 7.44(\mathrm{t}, J=7.6 \mathrm{~Hz}, 1$ $\mathrm{H}), 7.37(\mathrm{t}, J=7.7 \mathrm{~Hz}, 4 \mathrm{H}), 7.29-7.14(\mathrm{~m}, 10 \mathrm{H}), 7.02-6.98$ $(\mathrm{m}, 2 \mathrm{H}) .{ }^{19} \mathrm{~F}$ NMR (471 MHz, methylene chloride- $\left.d_{2}\right): \delta=$ -106.75. ESI-ITMS: m/z: 573.13 [M ${ }^{+}$]. Calcd: 573.20. Elem. Anal. Calcd. (\%) for $\mathrm{C}_{39} \mathrm{H}_{25} \mathrm{~N}_{3} \mathrm{~F}_{2}$ : C, 81.66; $\mathrm{H}, 4.39 ; \mathrm{N}, 7.33$. Found: C, 81.28; H, 4.62; N, 7.29.

2FPPImTPA: 2b (1.94 g, $4 \mathrm{mmol}$ ) and (4-(diphenylamino)phenyl)boronic acid (1.27 g $4.4 \mathrm{mmol}$ ) were reacted according to Reaction II to give 2FPPImTPA. A white powder, mass: $2.31 \mathrm{~g}$, yield: $89 \% .{ }^{1} \mathrm{H}$ NMR $(500 \mathrm{MHz}$, methylene chloride- $\left.d_{2}\right): \delta=8.90-8.81(\mathrm{~m}, 2 \mathrm{H}), 8.77(\mathrm{~d}, J=8.3 \mathrm{~Hz}, 1$ $\mathrm{H}), 7.92(\mathrm{t}, J=1.8 \mathrm{~Hz}, 1 \mathrm{H}), 7.84-7.77(\mathrm{~m}, 1 \mathrm{H}), 7.72$ (ddd, $J=8.4,6.9,1.5 \mathrm{~Hz}, 1 \mathrm{H}), 7.65(\mathrm{dt}, J=7.8,1.6 \mathrm{~Hz}, 1 \mathrm{H}), 7.60$ (ddd, $J=8.3,6.9,1.3 \mathrm{~Hz}, 1 \mathrm{H}), 7.53(\mathrm{dt}, J=7.7,1.5 \mathrm{~Hz}, 1 \mathrm{H}$ ), 7.48-7.39 (m, $4 \mathrm{H}), 7.37-7.32$ (m, $6 \mathrm{H}), 7.27-7.15$ (m, $10 \mathrm{H})$, 7.13-7.09 (m, $2 \mathrm{H}) .{ }^{19} \mathrm{~F}$ NMR (471 MHz, methylene chloride$\left.d_{2}\right): \delta=-106.61$. ESI-ITMS: $m / z: 649.17\left[\mathrm{M}^{+}\right]$. Calcd: 649.23. Elem. Anal. Calcd. (\%) for $\mathrm{C}_{45} \mathrm{H}_{29} \mathrm{~N}_{3} \mathrm{~F}_{2}$ : C, 83.19; $\mathrm{H}$, 4.50; N, 6.47. Found: C, 82.95; H, 4.70; N, 6.40.

2FPPITPA: 2a (1.94 g, $4 \mathrm{mmol}$ ) and (4-(diphenylamino) phenyl)boronic acid (1.27 g $4.4 \mathrm{mmol}$ ) were reacted according to Reaction II to give 2FPPITPA. A pale yellow powder, mass: 2.39 g, yield: $92 \% .{ }^{1} \mathrm{H}$ NMR (500 MHz, methylene chloride- $\left.d_{2}\right): \delta=8.87(\mathrm{t}, J=11.6 \mathrm{~Hz}, 2 \mathrm{H}), 8.79(\mathrm{~d}, J=8.3 \mathrm{~Hz}$, $1 \mathrm{H}), 7.84-7.79$ (m, $1 \mathrm{H}), 7.76-7.68$ (m, $3 \mathrm{H}$ ), 7.66-7.60 (m, 3 H), 7.58-7.55 (m, $2 \mathrm{H}), 7.43$ (ddd, $J=8.2,6.9,1.2 \mathrm{~Hz}, 1 \mathrm{H}$ ), 7.35-7.30 (m, $5 \mathrm{H}), 7.27-7.19$ (m, $3 \mathrm{H}), 7.19-7.14(\mathrm{~m}, 6 \mathrm{H})$, 7.13-7.07 (m, $2 \mathrm{H}) .{ }^{19} \mathrm{~F}$ NMR (471 MHz, methylene chloride$\left.d_{2}\right): \delta=-106.60$. ESI-ITMS: $m / z: 649.73\left[\mathrm{M}^{+}\right]$. Calcd: 649.23. Elem. Anal.Calcd.(\%) for $\mathrm{C}_{45} \mathrm{H}_{29} \mathrm{~N}_{3} \mathrm{~F}_{2}$ : $\mathrm{C}, 83.19 ; \mathrm{H}, 4.50 ; \mathrm{N}, 6.47$. Found: C, 82.97; H, 4.69; N, 6.41.

\section{Funding Information}

This work was supported by the National Natural Science Foundation of China (21935005 and 51803069) and Program for JLU Science and Technology Innovative Research Team (2019TD-33).

\section{Acknowledgment}

We thank the staff from BL17B beamline of National Facility for Protein Science in Shanghai (NFPS) at Shanghai Synchrotron Radiation Facility (SSRF) for assistance during crystal-data collection.

\section{Supporting Information}

Supporting information for this article is available online at: https://doi.org/10.1055/s-0039-3402513.

\section{Primary Data}

Crystal files of 2FPPImTPA, 2FPPIDPA, and 2FPPITPA are provided in CCDC 1939337, 1939338, and 1939339, respectively. Primary data for this article are available online at: https://doi.org/10.1055/s-0039-3402513 and can be cited using the following DOI: 10.4125/pd0112th.

\section{References}

(1) Zhu, M.; Yang, C. Chem. Soc. Rev. 2013, 42, 4963.

(2) (a) Tung, Y.-J.; Ngo, T.; Hack, M.; Brown, J.; Koide, N.; Nagara, Y.; Kato, Y.; Ito, H. SID Symp. Dig. Tech. Pap. 2004, 35, 48. (b) Lee, M. T.; Liao, C. H.; Tsai, C. H.; Chen, C. H. Adv. Mater. 2005, 17, 2493.

(3) Chan, C. Y.; Tanaka, M.; Nakanotani, H.; Adachi, C. Nat. Commun. 2018, 9, 5036.

(4) (a) Pershin, A.; Hall, D.; Lemaur, V.; Sancho-Garcia, J. C.; Muccioli, L.; Zysman-Colman, E.; Beljonne, D.; Olivier, Y. Nat. Commun. 2019, 10, 597. (b) Reineke, S. Nat. Photonics 2014, 8, 269.

(5) (a) Yao, L.; Yang, B.; Ma, Y. G. Sci. China Chem. 2014, 57, 335. (b) Xu, Y.; Wang, C.; Zhou, X.; Zhou, J.; Guo, X.; Liang, X.; Hu, D.; Li, F.; Ma, D.; Ma, Y. J. Phys. Chem. Lett. 2019, 10, 6878.

(6) Li, W. J.; Liu, D. D.; Shen, F. Z.; Ma, D. G.; Wang, Z. M.; Feng, T.; Xu, Y. X.; Yang, B.; Ma, Y. G. Adv. Funct. Mater. 2012, 22, 2797. 
(7) Zhang, S.; Li, W.; Yao, L.; Pan, Y.; Shen, F.; Xiao, R.; Yang, B.; Ma, Y. Chem. Commun. 2013, 49, 11302.

(8) Zhang, S.; Yao, L.; Peng, Q.; Li, W.; Pan, Y.; Xiao, R.; Gao, Y.; Gu, C.; Wang, Z.; Lu, P.; Li, F.; Su, S.; Yang, B.; Ma, Y. Adv. Funct. Mater. 2015, 25, 1755.

(9) Xu, Y.; Liang, X.; Zhou, X.; Yuan, P.; Zhou, J.; Wang, C.; Li, B.; Hu, D.; Qiao, X.; Jiang, X.; Liu, L.; Su, S. J.; Ma, D.; Ma, Y. Adv. Mater. 2019, 31, 1807388 .

(10) (a) Ouyang, X.; Li, X. L.; Ai, L.; Mi, D.; Ge, Z.; Su, S. J. ACS. Appl. Mater. Interfaces 2015, 7, 7869. (b) Cao, C.; Chen, W.-C.; Tian, S.; Chen, J.-X.; Wang, Z.-Y.; Zheng, X.-H.; Ding, C.-W.; Li, J.-H.; Zhu, J.-J.; Zhu, Z.-L.; Tong, Q.-X.; Lee, C.-S. Mater. Chem. Front. 2019, 3, 1071. (c) Cao, C.; Chen, W. C.; Chen, J. X.; Yang, L.; Wang, X. Z.; Yang, H.; Huang, B.; Zhu, Z. L.; Tong, Q. X.; Lee, C. S. ACS. Appl. Mater. Interfaces 2019, 11, 11691. (d) Chen, L.; Zhang, S.; Li, H.; Chen, R.; Jin, L.; Yuan, K.; Li, H.; Lu, P.; Yang, B.; Huang, W. J. Phys. Chem. Lett. 2018, 9, 5240.

(11) (a) Jo, J. W.; Jung, J. W.; Jung, E. H.; Ahn, H.; Shin, T. J.; Jo, W. H. Energy Environ. Sci. 2015, 8, 2427. (b) Priimagi, A.; Cavallo, G.; Metrangolo, P.; Resnati, G. Acc. Chem. Res. 2013, 46, 2686.

(12) Li, W.; Pan, Y.; Yao, L.; Liu, H.; Zhang, S.; Wang, C.; Shen, F.; Lu, P.; Yang, B.; Ma, Y. Adv. Opt. Mater. 2014, 2, 892.

(13) Lu, T.; Chen, F. J. Comput. Chem. 2012, 33, 580.

(14) El-Sayed, M. A. J. Chem. Phys. 1963, 38, 2834.

(15) Yuan, W. Z.; Shen, X. Y.; Zhao, H.; Lam, J. W. Y.; Tang, L.; Lu, P.; Wang, C.; Liu, Y.; Wang, Z.; Zheng, Q.; Sun, J. Z.; Ma, Y.; Tang, B. Z. J. Phys. Chem. C. 2010, 114, 6090. (b) Li, M.; Ling, K.; Shi, H.; Gan,
N.; Song, L.; Cai, S.; Cheng, Z.; Gu, L.; Wang, X.; Ma, C.; Gu, M.; Wu, Q.; Bian, L.; Liu, M.; An, Z.; Ma, H.; Huang, W. Adv. Opt. Mater. 2019, 7, 1800820.

(16) Liu, H.; Bai, Q.; Yao, L.; Zhang, H.; Xu, H.; Zhang, S.; Li, W.; Gao, Y.; Li, J.; Lu, P.; Wang, H.; Yang, B.; Ma, Y. Chem. Sci. 2015, 6, 3797.

(17) Mei, J.; Leung, N. L.; Kwok, R. T.; Lam, J. W.; Tang, B. Z. Chem. Rev. 2015, 115, 11718.

(18) Fan, D.; Yi, Y.; Li, Z.; Liu, W.; Peng, Q.; Shuai, Z. J. Phys. Chem. A. 2015, 119, 5233.

(19) (a) Li, C. L.; Li, Z. Q.; Liang, J. X.; Luo, H.; Liu, Y.; Wei, J. B.; Wang, Y. J. Mater. Chem. C. 2018, 6, 12888. (b) Li, Z.; Li, C.; Xu, Y.; Xie, N.; Jiao, X.; Wang, Y. J. Phys. Chem. Lett. 2019, 10, 842.

(20) (a) Wu, C. C.; Lin, Y. T.; Wong, K. T.; Chen, R. T.; Chien, Y. Y. Adv. Mater. 2004, 16, 61. (b) Kuwabara, Y.; Ogawa, H.; Inada, H.; Noma, N.; Shirota, Y. Adv. Mater. 1994, 6, 677.

(21) Tang, X.; Bai, Q.; Peng, Q.; Gao, Y.; Li, J.; Liu, Y.; Yao, L.; Lu, P.; Yang, B.; Ma, Y. Chem. Mater. 2015, 27, 7050.

(22) Chen, W. C.; Huang, B.; Ni, S. F.; Xiong, Y.; Rogach, A. L.; Wan, Y.; Shen, D.; Yuan, Y.; Chen, J. X.; Lo, M. F.; Cao, C.; Zhu, Z. L.; Wang, Y.; Wang, P.; Liao, L. S.; Lee, C. S. Adv. Funct. Mater. 2019, 29, 1903112.

(23) Chiang, C.-J.; Kimyonok, A.; Etherington, M. K.; Griffiths, G. C.; Jankus, V.; Turksoy, F.; Monkman, A. P. Adv. Funct. Mater. 2013, 23, 739.

(24) Brutting, W.; Frischeisen, J.; Schmidt, T. D.; Scholz, B. J.; Mayr, C. Phys. Status Solidi A 2013, 210, 44.

(25) Baldo, M. A.; O'Brien, D. F.; Thompson, M. E.; Forrest, S. R. Phys. Rev. B: Condens. Matter 1999, 60, 14422. 